\title{
Surveillance des personnes ayant obtenu un résultat négatif au test de la COVID-19 en Ontario, du 22 janvier au 22 février 2020
}

\author{
Michelle Murti ${ }^{1,2 *}$, Michael Whelan ${ }^{1}$, Andrea Saunders ${ }^{1}$, Karin Hohenadel ${ }^{1}$, Jonathan Gubbay ${ }^{1,3}$, \\ Sarah Buchan ${ }^{1,2}$
}

\section{Résumé}

Depuis le 22 janvier 2020, la « maladie causée par un nouveau coronavirus » est devenue une maladie à déclaration obligatoire d'importance pour la santé publique en Ontario. Les bureaux de santé publique ont reçu des directives sur la saisie des patients testés pour le coronavirus responsable du syndrome respiratoire aigu sévère (SRAS-CoV-2), le virus causant la maladie à coronavirus 2019 (COVID-19), dans le système provincial d'information sur la santé publique. Entre le 22 janvier et le 22 février 2020, 359 personnes ont reçu un résultat négatif de test et trois cas de COVID-19 ont été confirmés. Parmi les personnes dont le résultat était négatif, $51 \%$ étaient des femmes et $71 \%$ avaient moins de 50 ans. Les symptômes les plus fréquemment signalés étaient la toux (55\%), la fièvre (37 \%) et le mal de gorge (35\%). La majorité des personnes ont été testées dans les trois jours suivant l'apparition des symptômes, mais plus d'un quart d'entre elles l'ont été plus de sept jours après. Durant le premier mois de déclaration, les antécédents de voyage déclarés ont passé de la Chine à une proportion croissante de voyages ailleurs qu'en Chine.
Cette oeuvre est mise à la disposition selon les termes de la licence internationale Creative Commons Attribution 4.0

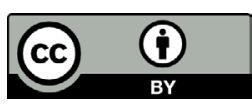

Affiliations

1 Santé publique Ontario, Toronto, ON

2 Dalla Lana School of Public Health, Université de Toronto, Toronto, ON

${ }^{3}$ Université de Toronto, Toronto, ON

\section{*Correspondance :}

michelle.murti@oahpp.ca

Citation proposée : Murti M, Whelan M, Saunders A, Hohenadel K, Gubbay J, Buchan S. Surveillance des personnes ayant obtenu un résultat négatif au test de la COVID-19 en Ontario, du 22 janvier au 22 février 2020. Relevé des maladies transmissibles au Canada 2020;46(5):170-4. https://doi.org/10.14745/ccdr.v46i05a08f Mots-clés : coronavirus, COVID-19, surveillance, Ontario, dépistage

\section{Introduction}

En décembre 2019, le syndrome clinique causé par le coronavirus responsable du syndrome respiratoire aigu sévère 2 (SRAS CoV 2) (maladie à coronavirus 2019, COVID-19) a été identifié après une éclosion de maladie de type pneumonie à Wuhan, en Chine (1). L'identification rapide du virus à l'origine de l'éclosion et la mise au point de méthodes de dépistage ont permis aux pays du monde entier de tester et d'identifier les cas à l'intérieur de leurs frontières (2). Au début de l'épidémie en Chine, l'Ontario a alerté les fournisseurs de soins de santé et recommandé de tester les personnes ayant des antécédents de voyage à Wuhan, en Chine. Depuis le 22 janvier 2020, la « maladie causée par un nouveau coronavirus » est considérée comme une maladie à déclaration obligatoire d'importance pour la santé publique en Ontario, et comprend des définitions de cas pour les personnes faisant l'objet d'une enquête (POE) et pour les cas probables, présumés et confirmés $(3,4)$. En Ontario, les bureaux de santé publique locaux sont chargés de recevoir la notification des personnes faisant l'objet d'une enquête soumises à un test de dépistage de la COVID-19, et de fournir des conseils sur la gestion de la santé publique des personnes soumises au test (5). Le 28 janvier 2020, Santé publique Ontario a publié des directives à l'intention des bureaux de santé sur l'utilisation du Système d'information sur la santé publique intégré (SISP-i) pour saisir les renseignements sur les personnes faisant l'objet d'une enquête et sur les cas. Par la suite, la COVID-19 s'est propagée dans le monde entier et a fait l'objet de multiples introductions en Ontario et dans d'autres régions du Canada (6), le premier cas en Ontario ayant été signalé le 25 janvier.

Notre objectif est de décrire les données de surveillance de la santé publique sur les personnes qui ont été déclarées à la santé publique et qui ont ensuite reçu un résultat négatif du test de la COVID-19, durant le premier mois après le début de la déclaration obligatoire en Ontario (du 22 janvier au 22 février 2020).

\section{Situation : janvier à février 2020}

En raison de l'évolution rapide de l'épidémiologie mondiale et de la compréhension de la COVID-19, la définition de cas d'une personne faisant l'objet d'une enquête a été mise à jour à plusieurs reprises (tableau 1), afin d'élargir les zones touchées pour inclure toute la Chine continentale et d'assouplir les critères 
Tableau 1 : Définitions de cas pour les personnes faisant l'objet d'une enquête en Ontario, du 22 janvier au 22 février 2020

\section{Date applicable \\ Case definition version}

Une personne souffrant de fièvre et d'une maladie respiratoire aiguë ou d'une pneumonie ET répondant à l'un des critères suivants :

- Voyage à Wuhan, en Chine, dans les 14 jours précédant le début de la maladie OU

22 au 27 janvier 2020

- Contact étroit ${ }^{\mathrm{a}}$ avec un cas confirmé ou probable de COVID-19

OU

- Contact étroit avec une personne atteinte d'une maladie respiratoire aiguë qui s'est rendue à Wuhan, en Chine, dans les 14 jours précédant le début de sa maladie

Une personne souffrant de fièvre et d'une maladie respiratoire aiguë ou d'une pneumonie ET répondant à l'un des critères suivants :

- Voyage dans la province du Hubei, en Chine, dans les 14 jours précédant le début de la maladie

28 au 30 janvier 2020

OU

- Contact étroit avec un cas confirmé ou probable de COVID-19

OU

- Contact étroit avec une personne atteinte d'une maladie respiratoire aiguë qui s'est rendue dans la province du Hubei, en Chine, dans les 14 jours précédant le début de sa maladie

Une personne ayant de la fièvre et/ou de la toux ou des difficultés à respirer ET répondant à l'un des critères suivants :

- Voyage dans la province du Hubei, en Chine, dans les 14 jours précédant le début de la maladie

31 janvier au 7 février 2020

OU

- Contact étroit avec un cas confirmé ou probable de COVID-19

$$
\text { OU }
$$

- Contact étroit avec une personne atteinte d'une maladie respiratoire aiguë qui s'est rendue dans la province du Hubei, en Chine, dans les 14 jours précédant le début de sa maladie

Une personne ayant de la fièvre et/ou de la toux ou des difficultés à respirer ET répondant à l'un des critères suivants :

- Voyage en Chine continentale dans les 14 jours précédant le début de la maladie

OU

8 au 26 février 2020

- Contact étroit avec un cas confirmé ou probable de COVID-19

OU

- Contact étroit avec une personne atteinte d'une maladie respiratoire aiguë qui s'est rendue en Chine continentale dans les 14 jours précédant le début de sa maladie

Un contact étroit est défini comme une personne qui a fourni des soins au patient, y compris les travailleurs de la santé, les membres de la famille ou d'autres aidants naturels, ou qui a eu un autre contact physique étroit semblable OU qui a vécu avec un cas probable ou confirmé ou qui a eu un contact étroit et prolongé avec un cas probable ou confirmé pendant que le cas était malade relatifs aux symptômes. Les directives cliniques sur les indications pour effectuer le dépistage ont également évolué et, depuis le 22 février, tous les tests de laboratoire initiaux sont effectués à Santé publique Ontario $(5,7)$. Au cours de cette période, les résultats positifs et négatifs des tests de dépistage du SRAS-CoV-2 ont été communiqués au médecin-hygiéniste local.

Nous avons examiné les enregistrements de COVID-19 déclarés dans le Système d'information sur la santé publique intégré entre le 22 janvier et le 22 février 2020. Nous avons exclu les personnes répondant à la définition de cas provincial confirmé, présumé confirmé ou probable. Ces enregistrements portaient sur les personnes répondant à la définition de cas provinciale d'une personne faisant l'objet d'une enquête au moment de la déclaration, les personnes auprès desquelles le bureau de santé publique local assurait un suivi et les personnes qui avaient subi un test de dépistage de la COVID-19 déclaré au bureau de santé publique local $(n=466)$. Les enregistrements sans données de laboratoire dans le Système d'information sur la santé publique intégré ont été exclus des analyses, laissant 359 enregistrements dans notre ensemble de données. Nous avons évalué les expositions, les caractéristiques, les symptômes et le temps entre l'apparition des symptômes et les tests de ces personnes. Les expositions ont été classées selon une hiérarchie de voyages en Chine, de voyages hors de Chine et d'exposition en Ontario. Toutes les analyses ont été réalisées selon le Enterprise Guide de SAS, v.7.1 (SAS Institute Inc., Cary, Caroline du Nord).

Le nombre de personnes ayant obtenu un résultat négatif du test a atteint un sommet à la mi-février, avec 29 personnes déclarées le 10 février 2020 (figure 1). Ce sont les voyages en Chine qui ont été signalés par le plus grand nombre de patients ( $n=196,54,6 \%$ ) tout au long de la période d'étude. Après le 2 février 2020, 32 patients ont déclaré avoir voyagé en dehors de la Chine.

Figure 1 : Nombre de personnes ayant obtenu un résultat négatif du test de dépistage de la COVID-19 $(\mathrm{N}=359)$, par date de déclaration au bureau de santé publique et lieu d'exposition

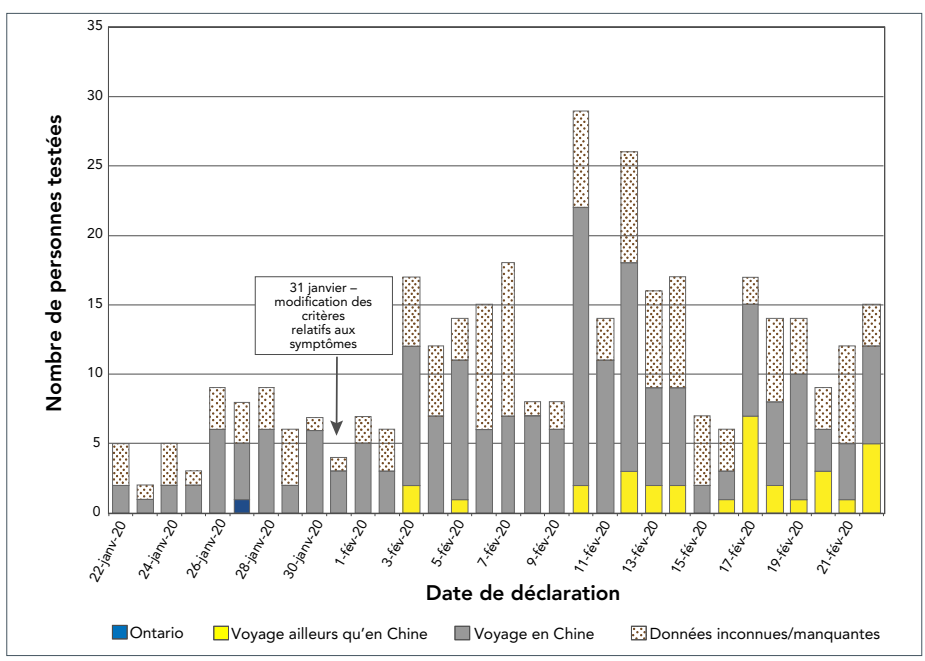


Un peu plus de la moitié $(51,8 \%)$ de ces patients étaient des femmes. La majorité $(71,3 \%)$ des patients avaient moins de 50 ans. Le plus grand nombre de patients testés ( $n=97,27,0 \%$ ) avaient entre 20 et 29 ans. Parmi les moins de 10 ans, 70,5\% étaient de sexe masculin (tableau 2). Les symptômes les plus fréquemment signalés parmi ceux pour lesquels les données ont été enregistrées $(n=314)$ sont la toux, la fièvre et le mal de gorge (tableau 3).

Tableau 2 : Âge et sexe des personnes ayant reçu un résultat négatif du test de dépistage de la COVID-19 $(\mathrm{N}=359)$

\begin{tabular}{|l|r|r|r|r|}
\hline $\begin{array}{l}\text { Groupe } \\
\text { d'âge } \\
\text { Moins de } \\
\text { dix ans }\end{array}$ & $\begin{array}{c}\text { Femmes } \\
(n=186)\end{array}$ & $\begin{array}{c}\text { Hommes } \\
(n=168)\end{array}$ & $\begin{array}{c}\text { Sexe non } \\
\text { précisé testéa } \\
(n=5)\end{array}$ & $\begin{array}{c}\text { Total du } \\
\text { groupe } \\
\mathbf{d}^{\prime} \text { âge } \\
(\mathrm{N}=359)\end{array}$ \\
\hline 10 à 19 ans & 6 & 31 & 0 & 44 \\
\hline 20 à 29 ans & 56 & 40 & 1 & 15 \\
\hline 30 à 39 ans & 32 & 23 & 1 & 97 \\
\hline 40 à 49 ans & 25 & 18 & 2 & 57 \\
\hline 50 à 59 ans & 34 & 25 & 0 & 43 \\
\hline 60 à 69 ans & 16 & 16 & 0 & 59 \\
\hline 70 à 79 ans & 1 & 6 & 0 & 32 \\
\hline 80 à 89 ans & 3 & 1 & 0 & 5 \\
\hline
\end{tabular}

Tableau 3 : Symptômes signalés par les personnes ayant reçu un résultat négatif du test de dépistage de la COVID-19 $(n=314)$

\begin{tabular}{|l|r|r|}
\multicolumn{1}{|c|}{ Symptôme } & $\begin{array}{c}\text { Nombre } \\
\text { d'enregistrements }\end{array}$ & $\begin{array}{c}\text { Pourcentage de } \\
\text { patients déclarant } \\
\text { le symptôme }\end{array}$ \\
\hline Toux & 171 & $54,5 \%$ \\
\hline Fièvre & 115 & $36,6 \%$ \\
\hline Mal de gorge & 109 & $34,7 \%$ \\
\hline Douleur & 58 & $18,5 \%$ \\
\hline Essoufflement & 47 & $15,0 \%$ \\
\hline Écoulement nasal & 35 & $11,1 \%$ \\
\hline Frissons & 21 & $6,7 \%$ \\
\hline Diarrhée & 20 & $6,4 \%$ \\
\hline Nausées & 7 & $2,2 \%$ \\
\hline Faiblesse & 1 & $0,3 \%$ \\
\hline a Nous ne disposions pas d'informations sur les symptômes pour tous les cas
\end{tabular}

Parmi les patie nts dont la date de prélèvement des échantillons se situe dans les 30 jours suivant l'apparition des symptômes ( $n=291 / 359,81,1 \%), 177(60,8 \%$ ) et $210(72,2 \%)$ ont subi le prélèvement dans les trois et sept jours suivant l'apparition des symptômes, respectivement. Cependant, 81 patients (27,8 \%) ont subi le prélèvement au moins sept jours après l'apparition des symptômes et 23 (7,9 \%) entre 14 et 30 jours après l'apparition des symptômes (figure 2).

Figure 2 : Nombre de jours entre la date de l'épisode et le prélèvement $(n=291)^{a}$

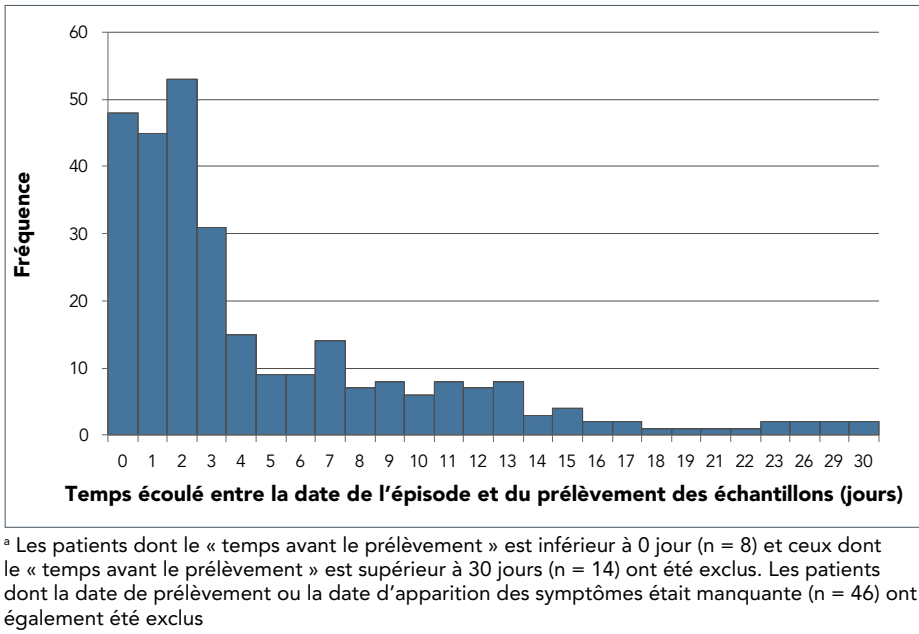

\section{Discussion}

Au cours du premier mois suivant la déclaration obligatoire de la COVID-19, les bureaux de santé publique ont enregistré 359 personnes ayant obtenu des résultats négatifs du test dans le système de surveillance provincial. En comparaison, les Centers for Disease Control and Prevention des États-Unis ont présenté un rapport sur l'ensemble du pays au 23 février (8). Le 22 février, il n'y avait que trois cas confirmés de COVID-19 en Ontario, qui s'étaient tous récemment rendus à Wuhan, en Chine.

La grande majorité des personnes testées avaient des antécédents de voyage en Chine, comme on pouvait s'y attendre, puisque le risque d'exportation était initialement centré sur Wuhan, en Chine, et étant donné les critères d'exposition de la définition de cas d'une personne faisant l'objet d'une enquête. Des interdictions de voyager ont été imposées par la ville de Wuhan à partir du 23 janvier, et ont été progressivement étendues à d'autres régions de la Chine au début des célébrations du Nouvel An lunaire (25 janvier 2020) afin de ralentir la propagation du virus dans d'autres régions (9). Par la suite, à partir du début du mois de février, les personnes déclarant avoir voyagé en dehors de la Chine représentent une

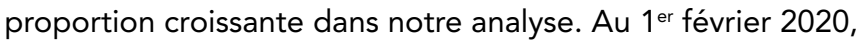
il y avait 7153 cas dans la province du Hubei, 11821 cas dans l'ensemble de la Chine et 132 cas dans 23 pays hors de Chine (10). Le 26 février, la définition de cas a été modifiée pour inclure d'autres zones touchées, ce qui a encore élargi la gamme des expositions signalées à la suite d'un voyage (données non présentées). 
Dans notre analyse, les personnes ayant un résultat négatif de test en Ontario étaient plus jeunes par rapport à la répartition par âge des cas confirmés signalés en Chine (11). Cependant, les groupes d'âge des personnes testées en Ontario étaient similaires à ceux des personnes faisant l'objet d'une enquête évaluées aux États-Unis (12). La structure par âge des personnes faisant l'objet d'une enquête en Ontario peut refléter les familles plus jeunes et les adultes en âge de travailler qui sont arrivés récemment de Chine ou qui sont revenus d'un séjour en Chine, par rapport aux adultes âgés qui ont peut-être moins tendance à voyager. Pour évaluer cette hypothèse, il faudrait disposer de renseignements sur les caractéristiques démographiques de tous les voyageurs de retour de Chine durant cette période.

Comme on pouvait s'y attendre d'après la définition de cas, la plupart des cas ont signalé de la toux ou de la fièvre, tout comme les personnes faisant l'objet d'une enquête et les cas dans les autres instances, où ce sont les deux symptômes les plus courants $(12,13)$. Le troisième symptôme le plus fréquent chez les personnes dont le test de dépistage est négatif en Ontario est le mal de gorge, déclaré par 34,7 \% des personnes pour lesquelles on dispose de données sur les symptômes. En comparaison, seuls 13,9\% des 55924 cas confirmés en laboratoire en Chine ont fait état d'un mal de gorge (13). En janvier et en février, des virus respiratoires saisonniers communs circulaient dans la communauté, ce qui pourrait expliquer la prévalence plus élevée des maux de gorge et pourrait fournir un autre diagnostic pour ces personnes $(14,15)$. Malgré l'absence d'inclusion des symptômes gastro-intestinaux dans la définition de cas, $6,4 \%$ des individus de notre analyse ont déclaré avoir la diarrhée. Des symptômes gastro-intestinaux ont également été signalés chez une minorité de patients atteints de la COVID-19 en Chine (13,16-19).

Il semble nécessaire de procéder à une évaluation et à un dépistage précoces, puisque la majorité des personnes de ce groupe d'étude se sont présentées pour le test dans les trois jours suivant l'apparition des symptômes. Cependant, environ un quart des personnes testées ont attendu plus de sept jours avant de subir le test. Avec seulement trois cas confirmés au cours de cette période, nous ne pouvons raisonnablement comparer les symptômes aux comportements de dépistage des personnes qui ont été infectées. Cependant, les retards dans les tests ont des répercussions sur le suivi effectué par la santé publique. Les dépistages tardifs peuvent ne pas avoir décelé le SRAS-CoV-2 si l'infection était déjà résolue chez les patients au moment où les tests ont été effectués. Les retards peuvent être dus à une forme légère de la maladie ou à une inquiétude accrue après l'apparition des symptômes, avec une prise de conscience mondiale croissante pendant cette période. Cela a des répercussions sur le suivi effectué par la santé publique si ces personnes reçoivent finalement un résultat positif de test étant donné les rapports de transmissions par des personnes en phase présymptomatique de la maladie $(20,21)$.
Le monde étant davantage sensibilisé à la COVID-19, il pourrait être utile de réévaluer le délai entre l'apparition des symptômes et le dépistage au fil du temps pour évaluer l'efficacité des messages de santé publique recommandant de s'isoler immédiatement et de demander des soins ou un test dès l'apparition des symptômes.

Nos conclusions s'accompagnent de plusieurs limites, qui sont inhérentes aux données de surveillance de la santé publique. Tout d'abord, les données du Système d'information sur la santé publique intégré sur les personnes testées dans la province sont incomplètes car les personnes ne sont pas signalées aux bureaux de santé publique locaux et en raison de la saisie sélective des personnes testées et de la saisie incomplète des résultats de laboratoire dans le Système. II se peut également que la saisie des symptômes et des antécédents de voyage soit incomplète selon qu'ils ont été recueillis auprès du prestataire de soins ou du patient. Enfin, il peut y avoir un écart dans le rappel des symptômes et de leur apparition chez les cas où le test a été effectué longtemps après l'apparition des symptômes.

\section{Conclusion}

Les résultats de notre surveillance montrent qu'en comparaison à d'autres instances, bon nombre des personnes identifiées au cours du premier mois suivant la déclaration obligatoire ont été soumises à des tests en Ontario, et que l'épidémiologie a évolué au fil du temps vers des expositions liées à des voyageurs ailleurs qu'en Chine. Il est nécessaire d'approfondir l'évaluation de l'importance relative du mal de gorge comme symptôme commun des personnes testées, alors qu'il a été moins souvent décrit parmi les cas en Chine. En outre, il convient d'analyser plus avant les raisons des retards importants constatés entre l'apparition des symptômes et le test chez certaines personnes, car ces retards peuvent avoir des répercussions importantes sur le suivi effectué par la santé publique.

\section{Déclaration des auteurs}

M. W. et A. S. ont analysé les données. K. H. a supervisé le projet. J. G. a examiné les données. M. M. et S. B. ont rédigé le manuscrit. Tous les co-auteurs ont contribué au manuscrit final.

\section{Conflit d'intérêts}

Tous les auteurs ont rempli et soumis le formulaire de I'International Committee of Medical Journal Editors (ICMJE) pour la divulgation des conflits d'intérêts potentiels. Aucun conflit d'intérêts potentiel n'a été révélé.

\section{Remerciements}

S. Menon, Santé publique Ontario, qui a assuré la mise en page du manuscrit. 


\section{Financement}

Ce travail a été appuyé par Santé publique Ontario.

\section{Références}

1. World Health Organization. Coronovirus disease (COVID-19) Pandemic. Geneva, Switzerland: WHO; 2020. https://www.who.int/ emergencies/diseases/novel-coronavirus-2019

2. World Health Organization. Coronavirus disease (COVID-19) technical guidance: Laboratory testing for 2019-nCoV in humans. Geneva, Switzerland: WHO; 2020. https://www. who.int/emergencies/diseases/novel-coronavirus-2019/ technical-guidance/laboratory-guidance

3. Ministry of Health. Infectious Diseases Protocol, Appendix A Disease-Specific Chapters. Chapter: Diseases caused by a novel coronavirus, including Severe Acute Respiratory Syndrome (SARS) and Middle East Respiratory Syndrome (MERS). Toronto, ON: Queen's Printer for Ontario; 2020. http://www.health.gov.on.ca/ en/pro/programs/publichealth/oph_standards/docs/coronavirus_ chapter.pdf

4. Ontario Ministry of Health and Ministry of Long-Term Care. Case Definition - Novel Coronavirus (COVID-19). Toronto, ON: Queen's Printer for Ontario; 2020. http://www.health.gov.on.ca/en/pro/ programs/publichealth/coronavirus/docs/2019_case_definition. pdf

5. Ministère de la Santé Ministère des Soins de longue durée Ontario. COVID-19 : Document d'orientation à l'intention du secteur de la santé. Toronto, ON : Imprimeur de la Reine pour l'Ontario; 2020. http://www.health.gov.on.ca/fr/pro/programs/publichealth/ coronavirus/2019_guidance.aspx

6. Agence de la santé publique Canada. Maladie à coronavirus (COVID-19) : Mise à jour sur l'éclosion. Ottawa, ON : Gouvernement du Canada; 2020. https://www.canada.ca/fr/sante-publique/ services/maladies/2019-nouveau-coronavirus.html

7. Ontario Agency for Health Protection and Promotion (Public Health Ontario). Coronavirus Disease 2019 (COVID-19) Testing. Toronto, ON: Queen's Printer for Ontario; 2020. https://www. publichealthontario.ca/en/laboratory-services/test-informationindex/wuhan-novel-coronavirus

8. Jernigan DB; CDC COVID-19 Response Team. Update: Public Health Response to the Coronavirus Disease 2019 Outbreak - United States, February 24, 2020. MMWR Morb Mortal Wkly Rep 2020;69(8):216-9. DOI PubMed

9. Qin A, Wang V. Wuhan, Center of Coronavirus Outbreak, is Being Cut Off by Chinese Authorities. The New York Times. Manhattan, NY: The New York Times Company; 2020. https://www.nytimes. com/2020/01/22/world/asia/china-coronavirus-travel.html

10. World Health Organization. Novel Coronavirus (2019nCoV) Situation Report - 12. Geneva, Switzerland: WHO: 2020. https://www.who.int/docs/default-source/ coronaviruse/situation-reports/20200201-sitrep-12-ncov. pdf?sfvrsn=273c5d35_2

11. [Novel Coronavirus Pneumonia Emergency Response Epidemiology Team]. [The epidemiological characteristics of an outbreak of 2019 novel coronavirus diseases (COVID-19) in China]. [Chin J Epidemiol] 2020:41(2):145-51. PubMed
12. Bajema $K L$, Oster $A M, M c G o v e r n ~ O L$, Lindstrom $S$, Stenger $M R$, Anderson TC, Isenhour C, Clarke KR, Evans ME, Chu VT, Biggs HM, Kirking HL, Gerber SI, Hall AJ, Fry AM, Oliver SE; 2019-nCoV Persons Under Investigation Team; 2019-CoV Persons Under Investigation Team. Persons Evaluated for 2019 Novel Coronavirus - United States, January 2020. MMWR Morb Mortal Wkly Rep 2020;69(6):166-70. DOI PubMed

13. World Health Organization. Report of the WHO-China Joint Mission on Coronavirus Disease 2019 (COVID-19). Geneva, Switzerland: WHO; 2020. https://www.who.int/docs/default-source/ coronaviruse/who-china-joint-mission-on-covid-19-final-report. pdf

14. Agence de la protection et la promotion de la santé (Santé publique Ontario). Bulletin sur les pathogènes respiratoires en Ontario. Toronto, ON: Imprimeur de la Reine pour l'Ontario; 2020. https://www.publichealthontario.ca/fr/data-and-analysis/ infectious-disease/respiratory-pathogens-weekly

15. Agence de la protection et la promotion de la santé (Santé publique Ontario). Rapports de surveillance des agents pathogènes des voies respiratoires. Toronto, ON : Imprimeur de la Reine pour l'Ontario; 2020. https://www.publichealthontario.ca/fr/data-and-analysis/ infectious-disease/laboratory-respiratory-pathogen-surveillance

16. Chen N, Zhou M, Dong X, Qu J, Gong F, Han Y, Qiu Y, Wang J, Liu $Y$, Wei Y, Xia J, Yu T, Zhang X, Zhang L. Epidemiological and clinical characteristics of 99 cases of 2019 novel coronavirus pneumonia in Wuhan, China: a descriptive study. Lancet 2020;395(10223):507-13. DOI PubMed

17. Huang $C$, Wang $Y$, Li X, Ren L, Zhao J, Hu Y, Zhang L, Fan G, Xu J, Gu X, Cheng Z, Yu T, Xia J, Wei Y, Wu W, Xie X, Yin W, Li H, Liu M, Xiao Y, Gao H, Guo L, Xie J, Wang G, Jiang R, Gao Z, Jin Q, Wang J, Cao B. Clinical features of patients infected with 2019 novel coronavirus in Wuhan, China. Lancet 2020;395(10223):497-506. DOI PubMed

18. Chan JF, Yuan S, Kok KH, To KK, Chu H, Yang J, Xing F, Liu J, Yip CC, Poon RW, Tsoi HW, Lo SK, Chan KH, Poon VK, Chan WM, Ip JD, Cai JP, Cheng VC, Chen H, Hui CK, Yuen KY. A familial cluster of pneumonia associated with the 2019 novel coronavirus indicating person-to-person transmission: a study of a family cluster. Lancet 2020;395(10223):514-23. DOl PubMed

19. Holshue $M L$, DeBolt $C$, Lindquist $S$, Lofy $K H$, Wiesman J, Bruce $H$ Spitters C, Ericson K, Wilkerson S, Tural A, Diaz G, Cohn A, Fox L, Patel A, Gerber SI, Kim L, Tong S, Lu X, Lindstrom S, Pallansch MA, Weldon WC, Biggs HM, Uyeki TM, Pillai SK; Washington State 2019-nCoV Case Investigation Team. Washington State 2019-nCoV Case Investigation Team. First case of 2019 novel coronavirus in the United States. N Engl J Med 2020;382(10):929-36.

DOI PubMed

20. Yu P, Zhu J, Zhang Z, Han Y, Huang L. A familial cluster of infection associated with the 2019 novel coronavirus indicating potential person-to-person transmission during the incubation period. $J$ Infect Dis. 2020;jiaa077. DOI PubMed

21. Huang R, Xia J, Chen Y, Shan C, Wu C. A family cluster of SARS-CoV-2 infection involving 11 patients in Nanjing, China. Lancet Infect Dis. 2020;S1473-3099(20)30147-X. DOI PubMed 